\title{
A NECESSARY CONDITION FOR AN EXTREMUM OF A DOUBLE INTEGRAL.
}

BY PROFESSOR MAX MASON.

(Read before the American Mathematical Society, April 28, 1906.)

LEGENDRE's necessary condition on a function $y=\phi(x)$ which minimizes or maximizes the integral

$$
\int_{x_{0}}^{x_{1}} f\left(x, y, y^{\prime}\right) d x
$$

is that the function $f_{y^{\prime} y^{\prime}}\left(x, \phi, \phi^{\prime}\right)$ must be positive or zero for a minimum, negative or zero for a maximum. There seems to have been no adequate treatment of double integrals in regard to the analogous condition.

Suppose the function $z=\phi(x, y)$ is of class $* D^{\prime}$ in a region $\Omega$, assumes the values $g$ on the boundary of $\Omega$, and renders the integral

$$
J=\iint_{\Omega} f(x, y, z, p, q) d x d y \quad\left(p=z_{x}, q=z_{y}\right)
$$

an extremum with respect to all functions of class $D^{\prime}$ which assume the values $g$ on the boundary. Let $\zeta(x, y)$ be an allowed variation, $i$. e., a function of class $D^{\prime}$ which vanishes on the boundary. Then the value of the integral

$$
J(\epsilon)=\iint_{\Omega} f\left(x, y, \phi+\epsilon \zeta, \phi_{x}+\epsilon \zeta_{x}, \phi_{y}+\epsilon \zeta_{y}\right) d x d y,
$$

where $\epsilon$ is a parameter, is a function of $\epsilon$ which has an extreme value for $\epsilon=0$. It follows $\dagger$ that $J^{\prime}(0)$ must vanish, and that $J^{\prime \prime}(0)$ must have the same sign or vanish, for all allowed variations $\zeta$. From the condition $J^{\prime}(0)=0$ is derived the dif-

* A function $u$ is of class $C^{(n)}$ in $\Omega$ when it is continuous within $\Omega$ and on the boundary, together with its derivatives of all orders less than or equal to $n$. If $u$ is continuous throughout $\Omega$, and if $\Omega$ may be divided into a finite number of sub-regions such that $u$ is of class $C^{(n)}$ in each closed sub-region, then $u$ is of class $D^{(n)}$ in $\Omega$. This notation is due to Bolza.

$\dagger$ The derivatives $J^{\prime}(\varepsilon)$ and $J^{\prime \prime}(\varepsilon)$ are continuous, under the assumption that $f$ is of class $C^{\prime \prime}$. 
ferential equation which $\phi$ must satisfy. The second condition states that the integral

$$
\delta^{2} J=\iint_{\Omega}\left\{\zeta_{x}^{2} f_{p p}+2 \zeta_{x} \zeta_{y} f_{p q}+\zeta_{y}^{2} f_{q q}+\zeta^{2} f_{z z}+2 \zeta \zeta_{x} f_{z p}+2 \zeta \zeta_{y} f_{z q}\right\} d x d y
$$

must have the same sign, or vanish, for all allowed variations ; the arguments of the derivatives of $f$ are $x, y, \phi, \phi_{x}, \phi_{y}$. It has been shown * that if

$$
\alpha^{2} f_{p p}+2 \alpha \beta f_{p q}+\beta^{2} f_{q q}
$$

is a positive definite form $\delta^{2} J$ is positive, if a negative definite form $\delta^{2} J$ is negative. In point of fact it is a necessary condition for either a minimum or a maximum that this form be at least semi-definite. This will be proved without transforming the second variation, $\delta^{2} J \cdot \dagger$

The theorem to be proved is then the following:

A necessary condition for an extremum is that

$$
f_{p p} f_{q q}-f_{p_{q}}^{2} \geqq 0
$$

at all points of $\Omega$.

Suppose, to the contrary, that the first member of (1) is negative at a point $\left(x_{0}, y_{0}\right)$ of $\Omega$. Then a region $R$ containing the point $\left(x_{0}, y_{0}\right)$ may be chosen, so small that throughout $R \phi_{x}$ and $\phi_{y}$ are continuous and

$$
f_{p p} f_{q q}-f_{p_{q}}^{2}<-m^{2}
$$

where $m$ is a fixed number. There exist, therefore, two real and distinct roots of the equation

$$
Q(h) \equiv f_{p p} h^{2}+2 f_{p q} h+f_{q q}=0
$$

for each point of $R . \quad Q(h)$ will have different signs according as $h$ lies between or outside these roots. Since the coefficients of $Q(h)$ are continuous within $R$, it follows that if $R$ be chosen $\$ 25$.

* See Kneser, Encyklopädie der Mathematischen Wissenschaften, II A8,

$\dagger$ The necessity of Legendre's condition in the case of simple integrals could be shown in a similar manner, without transforming the second variation. 
small enough, two fixed intervals $P$ and $N$ and a fixed number $\delta$ may be found, such that at all points of $R$

$$
\begin{aligned}
& f_{p p} h^{2}+2 f_{p q} h+f_{q q}>\quad \delta^{2} \text { for all } h \text { in } P, \\
& f_{p p} h^{2}+2 f_{p q} h+f_{q q}<\quad-\delta^{2} \text { for all } h \text { in } N .
\end{aligned}
$$

Choose for the region $R$ a rhombus, whose center is at $\left(x_{0}, y_{0}\right)$ and whose sides are formed by the lines

$$
\begin{array}{lll}
\text { (a) } u_{1}-\epsilon=0, & \text { (c) } u_{1}+\epsilon=0, \\
\text { (b) } u_{2}-\epsilon=0, & \text { (d) } u_{2}+\epsilon=0,
\end{array}
$$

where

$$
\begin{aligned}
& u_{1}=\left(x-x_{0}\right) \cos \alpha_{1}+\left(y-y_{0}\right) \sin a_{1}, \\
& u_{2}=\left(x-x_{0}\right) \cos a_{2}+\left(y-y_{0}\right) \sin \alpha_{2},
\end{aligned}
$$

the positive constant $\epsilon$ being chosen so small that (3) holds in $R$. The angles $a_{1}, \alpha_{2}$ will be determined later. The diagonals of the rhombus divide it into four triangles $a, \beta, \gamma, \delta$, containing the sides $a, b, c, d$, respectively. An allowed variation $\zeta$ is defined by the following equations, $\zeta$ being zero outside of $R$ :

Now

$$
\begin{array}{llll}
\zeta=-u_{1}+\epsilon & \text { in } a, & \zeta= & u_{1}+\epsilon \text { in } \gamma, \\
\zeta=-u_{2}+\epsilon & \text { in } \beta, & \zeta= & u_{2}+\epsilon \text { in } \delta .
\end{array}
$$

where

$$
\delta^{2} J=\delta_{1}^{2} J+\delta_{2}^{2} J,
$$

$$
\begin{gathered}
\delta_{1}^{2} J=\iint_{R} \zeta_{y}^{2}\left\{\left(\frac{\zeta_{x}}{\zeta_{y}}\right)^{2} f_{p p}+2 \frac{\zeta_{x}}{\zeta_{y}} f_{p q}+f_{q q}\right\} d x d y, \\
\delta_{2}^{2} J=\iint_{R}\left\{\zeta^{2} f_{z z}+2 \zeta \zeta_{x} f_{z p}+2 \zeta \zeta_{y} f_{z q}\right\} d x d y .
\end{gathered}
$$

Since $\left|\zeta_{x}\right| \leqq|1,| \zeta_{y}|\leqq 1,| \zeta \mid \leqq \epsilon$, it follows that

$$
\left|\delta_{2}^{2} J\right| \leqq \epsilon M A(\epsilon+4),
$$

where $M$ is the greatest of the maxima of $\left|f_{z z}\right|,\left|f_{z p}\right|,\left|f_{z q}\right|$, in $R$, and $A$ is the area of $R$. 
Now choose the angles $a_{1}$ and $a_{2}$ so that both of the quantities $\cot a_{1}$, cot $a_{2}$ lie either in the interval $P$ or in the interval $N$. Then the value of $\zeta_{x} / \zeta_{y}$ will lie either in $P$ or in $N$; if in $P \delta_{1}^{2} J$ will be positive, if in $N \delta_{1}^{2} J$ will be negative, on account of (3). In either case

$$
\left|\delta_{1}^{2} J\right|>\delta^{2} \iint_{R} \zeta_{y}^{2} d x d y=\delta^{2} \frac{A}{2}\left(\sin ^{2} \alpha_{1}+\sin ^{2} \alpha_{2}\right),
$$

where $A$ is the area of $R$. Therefore, from (4),

$$
\delta^{2} J>A\left[\frac{\delta^{2}}{2}\left(\sin ^{2} \alpha_{1}+\sin ^{2} \alpha_{2}\right)-\epsilon M(\epsilon+4)\right]
$$

if $\cot \alpha_{1}$ and $\cot \alpha_{2}$ lie in $P$, and

$$
\delta^{2} J<A\left[-\frac{\delta^{2}}{2}\left(\sin ^{2} \alpha,+\sin ^{2} \alpha_{2}\right)+\epsilon M(\epsilon+4)\right]
$$

if $\cot \alpha_{1}$ and $\cot \alpha_{2}$ lie in $N$. If $\epsilon$ be taken sufficiently small, $\delta^{2} J$ may therefore be made positive or negative at pleasure. This result, obtained under the supposition that the inequality (1) is not satisfied at some point in $\Omega$, contradicts the assumption that the function $\phi$ makes the integral $J$ an extremum. The condition (1) is therefore necessary for an extremum.

The above proof of the necessity of condition (1) depends upon a variation whose first derivatives have finite discontinuities. If the extremum problem be so stated that the functions $z$ are required to satisfy more restrictive conditions of continuity the proof must be altered, since the variations must satisfy these conditions also.

Suppose * the functions $z$ are required to be of class $C^{\prime \prime}$. For the region $R$, chosen in the previous case as a rhombus, take the region formed by the sub-regions $R_{1}, R_{2}, R_{3}$, defined in the following manner : $R_{1}$ is a rectangle having the sides

where

$$
u=\epsilon, \quad v=d, \quad u=-\epsilon, \quad v=-d,
$$

$$
\begin{gathered}
u=\left(x-x_{0}\right) \cos \alpha+\left(y-y_{0}\right) \sin \alpha, \\
v=\left(x-x_{0}\right) \sin \alpha-\left(y-y_{0}\right) \cos \alpha .
\end{gathered}
$$

* This is the assumption usually made in dealing with double integrals. 
The regions $R_{2}$ and $R_{3}$ are the portions exterior to $R_{1}$ of the areas bounded by the closed curves

$$
u^{2}+(v-d)^{4}-\epsilon^{2}=0, \quad u^{2}+(v+d)^{4}-\epsilon^{2}=0,
$$

respectively.

A variation $\zeta$ of class $C^{\prime \prime}$ is defined by the following equations, $\zeta$ being zero outside of $R$ :

Now

$$
\begin{array}{ll}
\zeta=\left(u^{2}-\epsilon^{2}\right)^{4} & \text { in } R_{1}, \\
\zeta=\left(u^{2}+(v-d)^{4}-\epsilon^{2}\right)^{4} & \text { in } R_{2}, \\
\zeta=\left(u^{2}+(v+d)^{4}-\epsilon^{2}\right)^{4} & \text { in } R_{3} .
\end{array}
$$

$$
\begin{aligned}
\delta^{2} J=\int & \int_{R_{1}} \zeta_{y}^{2}\left\{\left(\frac{\zeta_{x}}{\zeta_{y}}\right) f_{p p}+2 \frac{\zeta_{x}}{\zeta_{y}} f_{p q}+f_{q q}\right\} d x d y \\
& +\iint_{R_{1}+R_{2}}\left\{\zeta_{x}^{2} f_{p p}+2 \zeta_{x} \zeta_{y} f_{p q}+\zeta_{y}^{2} f_{q q}\right\} d x d y \\
& +\iint_{R}\left\{\zeta^{2} f_{z z}+2 \zeta \zeta_{x} f_{z p}+2 \zeta \zeta_{y} f_{z q}\right\} d x d y .
\end{aligned}
$$

Since $|u| \leqq \epsilon$ in $R,|v-d| \leqq \sqrt{\epsilon}$ in $R_{2}$ and $|v+d| \leqq \sqrt{\epsilon}$ in $R_{3}$, it follows that at all points of $R,\left|\zeta_{x}\right|<$ const. $\epsilon^{7},\left|\zeta_{y}\right|<$ const. $\epsilon^{7}$. Throughout $R,|\zeta| \leqq \epsilon^{8}$. The second and third integrands in the second member of the above equation are therefore numerically less than const. $\epsilon^{14}$ and const. $\epsilon^{15}$ respectively. The area of $R$ is less than $4(d+\sqrt{\epsilon}) \epsilon$, and the area of $R_{2}+R_{3}$ is less than $4 \epsilon^{\frac{3}{2}}$. Hence, for all values of $\epsilon$ sufficiently small,

$$
\left|\delta^{2} J-\iint_{R_{1}} \zeta_{y}^{2}\left\{\left(\frac{\zeta_{x}}{\zeta_{y}}\right)^{2} f_{p p}+2 \frac{\zeta_{x}}{\zeta_{y}} f_{p q}+f_{q q}\right\} d x d y\right|<K . \epsilon^{\frac{32}{2}},
$$

where $K$ is a constant. Now in $R_{1}$

$$
\zeta_{x} / \zeta_{y}=\cot \alpha .
$$

Therefore, if $\cot \alpha$ lies in the interval $P$, the integral in the first member of the above inequality is positive; if $\cot \alpha$ lies in $N$, it is negative. In either case, on account of (3), its 
absolute value is greater than

$$
\delta^{2} \iint_{R_{1}} \zeta_{y}^{2} d x d y=64 \delta^{2} \sin ^{2} a \int_{-d}^{d} \int_{-e}^{e}\left(u^{2}-\epsilon^{2}\right)^{6} u^{2} d u d v=c^{2} \epsilon^{15}
$$

where $c$ is a constant independent of $\epsilon$.

Therefore, if $\cot \alpha$ lies in the interval $P$,

$$
\delta^{2} J>\epsilon^{15}\left(c^{2}-K V^{\bar{\epsilon}}\right) .
$$

If $\cot \alpha$ lies in the interval $N$,

$$
\delta^{2} J<\epsilon^{15}\left(-c^{2}+K \sqrt{\epsilon}\right) .
$$

If $\epsilon$ be taken sufficiently small $\delta^{2} J$ may therefore be made positive or negative at pleasure, and the necessity of condition (1) follows at once.

Sheffield Scientific School, YALE UNIVERSITY.

\section{SHORTER NOTICES.}

Handbuch der Theorie der Gammafunktion. By Dr. Niels

Nrelsen, Docent in Mathematics at the University of Copenhagen. Leipzig, Teubner, 1906. 326 pages.

As the name indicates, this book is a comprehensive handbook of the theory of the gamma function; it contains nearly all the known formulas, gives very exhaustive references, and arranges the entire material into a systematic and readable whole. Besides a full index, a bibliography of over five hundred titles is appended to the volume, with exact citation including date and number of pages of each memoir. That Dr. Nielsen is fitted for the task which he had set himself is attested by the long list of his own contributions, exceeded only by those of Euler, Lerch and Schlömilch.

The book is divided into three parts : the first treats of series and the analytic theory, the second of the theory of the definite integral, and the third of the inverse problem of expanding other series in terms of gamma functions.

The first part (112 pages, 581 numbered formulas) com- 